\title{
Quality, not volume, determines outcome of coronary artery bypass surgery in a university-based community hospital network
}

\author{
Paul A. Kurlansky, MD, ${ }^{\mathrm{a}, \mathrm{b}}$ Michael Argenziano, MD, ${ }^{\mathrm{b}}$ Robert Dunton, MD, ${ }^{\mathrm{b}}$ Robert Lancey, MD, \\ Edward Nast, MD, ${ }^{\mathrm{b}}$ Allan Stewart, MD, ${ }^{\mathrm{b}}$ Timothy Williams, MD, ${ }^{\mathrm{b}}$ Alex Zapolanski, MD, ${ }^{\mathrm{b}}$ \\ Helena Chang, MS, ${ }^{\mathrm{c}}$ Judy Tingley, BSN, ${ }^{\mathrm{b}}$ and Craig R. Smith, $\mathrm{MD}^{\mathrm{b}}$
}

\begin{abstract}
Objectives: The present study examined the relationship between hospital and surgeon coronary artery bypass grafting procedural volume, mortality, morbidity, and National Quality Forum care processes in a universitybased community hospital quality improvement program.

Methods: The study population consisted of 2218 consecutive patients undergoing isolated coronary artery bypass grafting from 2007 to 2009 in a university-based quality improvement program that emphasizes involvement of all surgeons in the academic quality endeavor. The endpoints included operative mortality, major morbidity, and National Quality Forum-endorsed process measures as defined by the Society of Thoracic Surgeons. The procedural volume was analyzed as a categorical and continuous variable using general estimating equations, which accounted for clustering effects and which were adjusted for Society of Thoracic Surgeons risk scores and the propensity for operation in a low-versus high-volume program.
\end{abstract}

Results: The annual program volume ranged from 67 to 292 (median, 136; interquartile range, 88-224) and surgeon volume from 1 to 124 (median, 58; interquartile range, 30-89). The mortality rate among the hospitals was $0.47 \%$ to $2.23 \%$ ( $0.8 \%$ overall), and the observed/expected mortality ranged from 0 to 1.20 ( 0.41 overall). When comparing low-volume $(<200$ cases/year) and high-volume centers, no difference was found in the mortality (odds ratio [OR], 1.08; 95\% confidence interval [CI], 0.46-2.54, $P=.85$ ), morbidity (OR, 1.34; 95\% CI, 0.73-2.43), or any of the medication process measures. No difference was found in mortality (OR, $1.59 ; 95 \% \mathrm{CI}$, $0.81-3.13 ; P=.18)$, morbidity (OR, $1.20 ; 95 \% \mathrm{CI}, 0.86-1.66 ; P=.28)$, or medication failure (OR, $0.57,95 \%$ CI, $0.3-1.10 ; P=.10)$ between the high- and low-volume surgeons ( $<87)$. After adjustment for both the Society of Thoracic Surgeons risk score and the propensity score, no association was found for either hospital or surgeon volume with mortality or morbidity. However, a lack of compliance with National Quality Forum measures was highly predictive of morbidity $(\mathrm{OR}, 1.51 ; 95 \% \mathrm{CI}, 1.18-1.93 ; P=.001)$, regardless of volume, even after adjustment for predicted risk.

Conclusions: In the setting of a university-based community hospital quality improvement program, excellent surgical results can consistently be obtained even in relatively low-volume programs. The surgical outcomes were not associated with program or surgeon volume, but were directly correlated with the focus on quality as manifested by compliance with evidence-based quality standards. Meaningful university affiliation might represent a new quality paradigm for cardiac surgery in the community hospital setting. (J Thorac Cardiovasc Surg 2012;143:287-93)

\section{Supplemental material is available online.}

\footnotetext{
From the Florida Heart Research Institute, ${ }^{\mathrm{a}}$ Miami, Fla; and the Departments of Surgery ${ }^{\mathrm{b}}$ and Biostatistics, ${ }^{\mathrm{c}}$ Columbia University, New York, NY.

Disclosures: Authors have nothing to disclose with regard to commercial support.

Funded through an educational grant from the Department of Surgery, Columbia University, New York, NY.

Read at the 91st Annual Meeting of the American Association for Thoracic Surgery, Philadelphia, Pennsylvania, May 7-11, 2011.

Received for publication April 18, 2011; revisions received Sept 5, 2011; accepted for publication Oct 20, 2011; available ahead of print Nov 21, 2011.

Address for reprints: Paul A. Kurlansky, MD, Florida Heart Research Institute, 4770

Biscayne Boulevard, Suite 500, Miami FL 33140 (E-mail: doctorwu18@aol.com). 0022-5223/\$36.00

Copyright (c) 2012 by The American Association for Thoracic Surgery

doi:10.1016/j.jtcvs.2011.10.043
}

The evidence supporting a correlation between an increased case volume and decreased operative mortality for complex surgical procedures is copious and robust. ${ }^{1-3}$ In the case of coronary artery bypass grafting (CABG), the situation appears to be considerably more complex. ${ }^{4}$ A review of the aggregate data clearly seems to indicate that a similar trend, albeit perhaps less dramatic, exists for CABG surgery, just as for other complex operations. ${ }^{2,5,6}$ However, careful evaluation revealed a much more complicated picture. Recent analysis of the data from the Society of Thoracic Surgeons (STS) Adult Cardiac Database demonstrated that volume, although significantly correlated with mortality, accounted for only $1 \%$ of the composite quality score variation. ${ }^{7}$ Moreover, several careful studies have demonstrated an absence of a correlation between volume and mortality in CABG surgery. ${ }^{8-10}$ An analysis of the National 


$$
\begin{aligned}
& \text { Abbreviations and Acronyms } \\
& \begin{aligned}
\text { CABG } & =\text { coronary artery bypass grafting } \\
\text { CI } & =\text { confidence interval } \\
\text { HV } & =\text { high volume } \\
\text { LV } & =\text { low volume } \\
\text { OR } & =\text { odds ratio } \\
\text { STS } & =\text { Society of Thoracic Surgeons }
\end{aligned}
\end{aligned}
$$

Inpatient Sample revealed that $85 \%$ of "low-volume" (LV) and $89 \%$ of "medium-volume" hospital-years had riskstandardized mortality rates that were statistically lower or comparable to those expected, and only $6 \%$ of "highvolume" (HV) hospital-years had outcomes that were statistically better than expected. ${ }^{11}$ Therefore, many LV programs have excellent results, and some larger programs have less than average results. ${ }^{12}$ Thus, volume, although a readily available and easily trackable parameter, is neither a requirement nor a guarantee of quality.

During the past decade, an increase has occurred in cardiac surgical programs despite a marked decline in CABG volume.$^{13}$ Remarkably, however, mortality has continued to decline. ${ }^{14}$ Perhaps even more surprising is that this decrease in mortality has been achieved in the face of an increasingly complex, high-risk patient population. ${ }^{15}$ Clearly, the relation between volume and quality in CABG surgery is complex at best. We therefore decided to study the effect of surgical volume on the processes and outcomes of CABG surgery in a network of LV and medium-volume community hospital cardiac surgical programs affiliated with an academic medical center. It is our hypothesis that a concerted and informed focus on quality is more important than case volume in determining the outcomes of CABG surgery.

\section{MATERIALS AND METHODS \\ Study Population}

The study populations consisted of all patients who underwent isolated CABG surgery in any of five cardiac surgery programs affiliated with the Columbia University HeartSource program from 2007 to 2009. The Columbia HeartSource project represents the services of a team that includes senior cardiac surgeons and cardiologists, clinical staff, business managers, and administrators, all from the Divisions of Cardiothoracic Surgery and Cardiology of Columbia University Medical Center (available at: http:// www.columbiasurgery.org/cli/heartsrc/). The purpose of the project is to engage community hospitals in an academically-based endeavor to enhance an existing cardiovascular program or to launch one de novo. The program involves active involvement of the Columbia faculty in quality improvement, strategic planning, physician recruitment, regular morbidity/ mortality/quality rounds, external peer review of all deaths and other interesting or significant cases, and the availability of Columbia faculty for realtime consultation and discussion of challenging cases, academic teaching rounds, and opportunities for direct intraoperative teaching at both the Columbia Medical Center campus and the local level. All surgical cases are prospectively entered into a web-based proprietary STS-compliant database that is regularly monitored for timeliness and accuracy. Entry into the present study included all hospitals in the network that had been participating in the program for at least a full year before study inception in 2007 , and for at least 2 years of validated data were available. These criteria resulted in the review of five surgical programs from January 1, 2007 to December 31, 2009-one with 2 years of data and the remainder with 3. A total of 3341 patients had undergone cardiac surgery during the study period, of whom 2218 underwent isolated CABG. Because of the complexity of the potential confounding variables and the need to maintain adequate study volume, only patients undergoing isolated CABG were included in the present analysis.

There were several analysis-specific exclusion criteria. First, the patients with preoperative renal failure were excluded when analyzing postoperative renal failure. Second, the patients with preoperative cerebrovascular accidents were excluded when analyzing stroke. Third, the patients with previous $\mathrm{CABG}$ were excluded when analyzing internal thoracic artery use. Fourth, the patients who died in the hospital were excluded when analyzing discharge data. Fifth, the patients who had contraindications to any of the perioperative medications were excluded when analyzing medication compliance. Finally, the patients who died were excluded when analyzing the quality process measures.

\section{Primary Predictors}

The CABG volume was treated as a categorical variable. A hospital for a particular year was considered LV if it had fewer than 200 CABG cases. We also considered surgeon volume and hospital quality. The surgeons for a particular year were regarded as LV if they performed fewer than 59 CABG operations. In terms of quality, each patient received a "total quality score," ranging from 0 to 5, depending on the number of National Quality Forum measures that were achieved. A patient with a score of 5 was considered as receiving high-quality care from the hospital; otherwise, the patient received low-quality care.

\section{Study Endpoints}

The standard STS definitions were applied to all variables. The relation between hospital and surgeon CABG volume was correlated with those risk factors that were significant in the STS risk model. The endpoints included National Quality Forum-endorsed process measures (internal thoracic artery graft; preoperative $\beta$-blockade; and discharge $\beta$-blockade, antiplatelet agents, and lipid drugs), operative mortality (30 days), major morbidity (stroke, renal failure, reoperation, sternal infection, and prolonged ventilation). All calculations of operative or complication risk were computed using the STS risk calculator.

\section{Statistical Analyses}

Marginal logistic regression models based on generalized estimating equations were performed to examine the crude and adjusted relationships between CABG outcomes and hospital volume while accounting for correlation among patients within the same hospital. The propensity score methods were used to achieve a better balance in patient characteristics between LV and HV hospitals. The patients were separated into quintiles defined by their propensity scores, and the propensity quintile was included in the model already adjusted for hospital volume and predicted risk score. Generalized estimating equation methods were also applied to analyses that examined the effect of surgeon volume on CABG outcomes, accounting for correlation among patients treated by the same surgeon. Both crude and predicted risk score adjusted analyses were performed. Similarly, the generalized estimating equations approach was used to compare CABG outcomes between low- and high-quality patients, accounting for withinhospital associations. As before, crude and predicted risk score adjusted analyses were performed. A marginal log-linear regression model was fit to determine the relationship between a discrete quality score (ranging from 0 to 5) and hospital volume, accounting for hospital cluster. Odds 
TABLE 1. Selected baseline comparisons by hospital volume

\begin{tabular}{lccc}
\hline \multicolumn{1}{c}{ Characteristic } & $\begin{array}{c}\text { Low volume } \\
(\mathbf{n}=\mathbf{7 8 7})\end{array}$ & $\begin{array}{c}\text { High volume } \\
(\mathbf{n}=\mathbf{1 4 3 1})\end{array}$ & $\begin{array}{c}\boldsymbol{P} \\
\text { value }\end{array}$ \\
\hline Age (yr) & $64.6 \pm 10.4$ & $65.9 \pm 10.4$ & .004 \\
Male (n) & $593(75.3)$ & $1063(74.3)$ & .58 \\
Weight (kg) & $88.7 \pm 19.7$ & $87.1 \pm 18.3$ & .069 \\
Height (cm) & $171.1 \pm 10.3$ & $172.5 \pm 9.91$ & .002 \\
Diabetes (n) & $329 \pm 41.8$ & $490(34.2)$ & $<.001$ \\
Ejection fraction (\%) & $48.9 \pm 12.5$ & $52.6 \pm 11.5$ & $<.001$ \\
Last preoperative creatinine & $1.2(0.9)$ & $1.2(0.9)$ & .22 \\
$\quad$ level (n) & & & \\
Chronic lung disease (n) & $216(27.4)$ & $168(11.7)$ & $<.001$ \\
Hypertension (n) & $645(82)$ & $1191(83.2)$ & .45 \\
Peripheral arterial disease (n) & $131(16.6)$ & $165(11.5)$ & $<.001$ \\
Cerebrovascular disease (n) & $114(14.5)$ & $160(11.2)$ & .02 \\
Cardiogenic Shock (n) & $5(0.6)$ & $8(0.6)$ & .78 \\
Reoperation (n) & $17(2.2)$ & $32(2.2)$ & .91 \\
Nonelective status (n) & $504(64)$ & $666(46.5)$ & $<.001$ \\
\hline Dan & & &
\end{tabular}

Data in parentheses are percentages.

ratios (ORs) and $95 \%$ confidence intervals (CIs) were reported for all analyses. All analyses were performed using SAS, version 9.2 (SAS Institute, Cary, NC). $P<.05$ was considered significant.

\section{Missing Data}

No data were missing for the $\mathrm{CABG}$ outcomes or the primary predictors of interest. However, missing data were encountered when creating the propensity score model, which incorporated variables in the STS 2008 Risk Model that had a role in all the endpoints measured. We followed the imputation guidelines for continuous and categorical variables provided in the STS report (period ending December 31, 2009).

\section{Institutional Review Board}

Institutional review board approval was obtained from the Columbia University institutional board and appropriate review boards at each of the hospitals involved in the study.

\section{RESULTS}

The hospital volume/year ranged from 67 to 292 (median, 136; interquartile range, 88-224), with three hospitals consistently performing fewer than 200 cases/year (range, 67-140), hereafter referred to as LV hospitals, and two consistently performing more than 200 cases/year (range, 201-292), hereafter referred to as HV hospitals. The distribution of baseline characteristics between the LV and HV programs is listed in Table 1. Diabetes, reduced ejection fraction, chronic obstructive lung disease, peripheral arterial disease, cerebrovascular disease, and urgent or emergent status were all statistically more prevalent among the LV than the HV patients. Off-pump surgery was much more common in one of the two HV hospitals. Because this reflected a particular surgeon preference rather than a volume-related phenomenon, the effect of the off-pump versus on-pump preference could not be evaluated in the present study. Of those patients who underwent on-pump CABG, the bypass time was longer in the $\mathrm{LV}(117 \pm 4.4$
TABLE 2. Unadjusted odds ratios of outcome and quality measures for patients in low- and high-volume hospitals

\begin{tabular}{|c|c|c|}
\hline Variable & OR $(95 \% \mathrm{CI})$ & $P$ Value \\
\hline \multicolumn{3}{|l|}{ Domains } \\
\hline Mortality & $1.08(0.46-2.54)$ & .85 \\
\hline Morbidity & $1.34(0.73-2.43)$ & .34 \\
\hline ITA failure & $1.92(1.15-3.19)$ & .01 \\
\hline Medication failure & $0.57(0.25-1.30)$ & .18 \\
\hline \multicolumn{3}{|l|}{ Morbidity } \\
\hline Stroke & $0.32(0.21-0.49)$ & $<.001$ \\
\hline Reoperation & $1.01(0.51-2.00)$ & .98 \\
\hline Sternal infection & $4.13(2.16-7.86)$ & $<.001$ \\
\hline Prolonged ventilation & $1.75(1.01-3.03)$ & .048 \\
\hline Renal failure & $1.27(0.51-3.17)$ & .60 \\
\hline \multicolumn{3}{|l|}{ Medication failure } \\
\hline Preoperative $\beta$-blockade & $0.54(0.19-1.53)$ & .25 \\
\hline $\mathrm{D} / \mathrm{C} \beta$-blockade & $0.55(0.18-1.68)$ & .29 \\
\hline Antiplatelet agents & $0.80(0.48-1.33)$ & .39 \\
\hline Lipid drugs & $0.60(0.24-1.47)$ & .26 \\
\hline
\end{tabular}

$O R$, Odds ratio; $C I$, confidence interval; $I T A$, internal thoracic artery; $D / C$, hospital discharge.

minutes, least squares mean \pm standard error) than in the HV $(96.4 \pm 5.4)$ programs $(P=.033)$, but the crossclamp time (78.1 \pm 2.7 vs $72.7 \pm 3.4$ minutes; $P=.22)$ and number of distal grafts $(3.1 \pm 0.13$ vs $3.5 \pm 0.16 ; P=.075)$ was similar. The mortality rate among the hospitals ranged from $0 \%$ to $2.23 \%(0.8 \%$ overall $)$, and the annual observed/expected mortality ranged from 0 to 1.20 ( 0.41 overall). The total morbidity rate (sum of all five complications) ranged from $7.7 \%$ to $17.1 \%$ (11.7\% overall). Despite the somewhat different risk profile of the two groups, no difference was found in mortality or overall morbidity between the LV and HV groups (Table 2). A failure to use an internal thoracic artery graft was more common in the $\mathrm{LV}$ programs (OR, 1.92; 95\% CI, 1.15-3.19; $P=.01$ ), but no difference was found in the failure to meet any of the National Quality Forum medication parameters, either individually or collectively. Stroke appeared to be more common in the HV programs with a LV/HV OR of $0.32(95 \%$ CI, 0.21-0.49; $P<.001)$, and sternal infection was more common in the LV programs (OR, 4.13; 95\% CI, 2.16-7.86; $P<.001)$, although the latter result must be interpreted with some caution, because only six sternal wound infections developed during the entire study period. When each of those outcomes for which an STS risk score exists were compared after adjusting for predicted risk, stroke was still more common among the HV patients and sternal infection was still more common among the LV patients (Table 3 ). We further corrected for patient propensity to be treated at a LV versus HV program using propensity quintiles, while adjusting for predicted risk, and found that stroke was still more common amongst the HV patients and the incidence of sternal infection was no longer significantly different (Table E1). 
TABLE 3. Odds ratios for outcome measures in patients of low- and high-volume hospitals adjusted for predicted risk

\begin{tabular}{lcc}
\hline \multicolumn{1}{c}{ Variable } & OR $(\mathbf{9 5} \% \mathbf{C I})$ & $\boldsymbol{P}$ Value \\
\hline Mortality & $1.09(0.49-2.43)$ & .83 \\
Mortality or morbidity & $1.18(0.47-2.98)$ & .72 \\
Stroke & $0.33(0.22-0.50)$ & $<.001$ \\
Reoperation & $1.01(0.50-2.04)$ & .97 \\
Sternal infection & $3.51(1.95-6.31)$ & $<.001$ \\
Prolonged ventilation & $1.26(0.54-2.92)$ & .59 \\
Renal failure & $1.06(0.31-3.57)$ & .93 \\
\hline
\end{tabular}

$O R$, Odds ratio; $C I$, confidence interval.

We also analyzed the results in terms of surgeon rather than hospital volume. Surgeon volume/year ranged from 1 to 124 (median, 58.5; interquartile range, 59). When evaluating the outcomes for patients who had a LV $(<59$ cases/yr) versus a HV ( $\geq 59$ cases/yr) surgeon, we found no difference in mortality, morbidity, or any process measure, with the exception that the patients of a LV surgeon were less likely to receive preoperative $\beta$-blockade (OR, $0.64 ; 95 \%$ CI, $0.43-0.94 ; P=.02$; Table 4 ). When adjusted for predicted risk, no difference was seen in the incidence of mortality, overall, or any specific morbidity between patients of LV versus HV surgeons (Table E2).

Because compliance with process measures might represent a focus on quality, we assigned to each patient a simple "quality score" (range, 0-5) according to how many of the National Quality Forum measures were achieved for that given patient. Because the patient required successful discharge from the hospital for three of the five measures, all those patients who died in the hospital were excluded from this analysis. The vast majority of patients (1617/ $2195,73.7 \%$ ) had a $5 / 5$ score. A marginal log-linear regression analysis, accounting for within-hospital associations analyzing quality score as the outcome and hospital volume, failed to show an association $(P=.27)$. However, when the quality score $(5$ vs $<5)$ was correlated with morbidity, a statistically significant association was found with overall morbidity and with each individual complication, with the exception of sternal infection (Table 5). This association between process and outcome remained highly significant after adjusting for predicted risk.

\section{DISCUSSION}

Although evidence of an association between volume and mortality for CABG has been shown, the nature of this relationship is not clear. Evidence seems to contradict both the "practice makes perfect" intuitive approach, with smaller programs not uncommonly outperforming larger ones, ${ }^{11,12}$ and the "selective referral" phenomenon, with the higher volume hospitals and surgeons in New York State caring for the higher risk patients. ${ }^{5}$ With volume having so little predictive value in determining the outcomes of CABG patients, ${ }^{10}$ there clearly must be other
TABLE 4. Odds ratios of outcome measures for patients with lowversus high-volume surgeons

\begin{tabular}{lcc}
\hline \multicolumn{1}{c}{ Variable } & OR $(\mathbf{9 5} \% \mathbf{C I})$ & $\boldsymbol{P}$ Value \\
\hline Domains & & \\
$\quad$ Mortality & $1.59(0.81-3.13)$ & .18 \\
Morbidity & $1.20(0.86-1.66)$ & .28 \\
ITA failure & $1.80(0.65-4.96)$ & .26 \\
$\quad$ Medication failure & $0.57(0.30-1.10)$ & .10 \\
Morbidity & & \\
Stroke & $0.43(0.14-1.34)$ & .15 \\
Reoperation & $1.12(0.58-2.16)$ & .74 \\
Sternal infection & $0.66(0.11-3.86)$ & .64 \\
Prolonged ventilation & $1.26(0.83-1.91)$ & .28 \\
$\quad$ Renal failure & $0.91(0.47-1.77)$ & .78 \\
Medication failure & & \\
$\quad$ Preoperative $\beta$-blockade & $0.64(0.43-0.94)$ & .02 \\
D/C $\beta$-blockade & $0.70(0.46-1.08)$ & .10 \\
Antiplatelet agents & $0.59(0.25-1.41)$ & .24 \\
Lipid drugs & $0.81(0.24-2.68)$ & .73 \\
\hline OR, Odds ratio; $C I$, confidence interval; ITA, internal thoracic artery; $D / C$, hospital \\
discharge.
\end{tabular}

more compelling factors. Fortunately, as a profession, the investment that cardiac surgeons have made in developing robust registries with clinically meaningful risk adjustment permits measurement of outcomes directly rather than through surrogate markers. From the standpoint of public policy, this information facilitates knowledgeable decisions using actual, rather than surrogate, benchmarks. For the clinician, the challenge is to learn which factors appear to drive quality outcomes, and how (and whether) they can be more broadly applied into varied practice settings. We evaluated the outcomes of CABG surgery in a network of community hospitals affiliated in an active manner with a major academic medical center. The absence of correlation between surgical volume and outcome in such a small sampling of hospitals was not surprising, especially given the overall excellent results. However, what was compelling is that within this same patient cohort, the association between process measure and outcome was so strong.

Several points warrant discussion. First, the experience of decreasing mortality for CABG surgery in response to mandatory reporting has been somewhat universally observed. ${ }^{16,17}$ Second, concerted quality improvement efforts, assisted by well-organized clinical registries, has proved successful in multiple settings in improving outcomes. ${ }^{18-20}$ Third, although the application of process measures in various areas of medicine has had variable results in affecting outcomes, evidence is mounting that the adherence to quality measures is associated with improved mortality rates in CABG surgery, independent of hospital or surgeon volume. ${ }^{21}$ However, what is notable and somewhat unique in the present study is the correlation of process measures with outcomes that are totally unrelated to the specific processes addressed. Evidence is 
TABLE 5. Odds ratios of complications for patients with low- versus high-quality score

\begin{tabular}{llccc}
\hline & \multicolumn{2}{c}{ Unadjusted } & \multicolumn{2}{c}{ Adjusted for predicted score } \\
\cline { 2 - 4 } \multicolumn{1}{c}{ Variable } & OR $(\mathbf{9 5} \% \mathbf{C I})$ & $\boldsymbol{P}$ value & ORs $(\mathbf{9 5} \% \mathbf{C I})$ & \\
\hline Morbidity & $1.79(1.38-2.31)$ & $<.001$ & & \\
Stroke & $1.60(1.27-2.02)$ & $<.001$ & $1.51(1.18-1.93)$ & .001 \\
Reoperation & $1.88(1.44-2.46)$ & $<.001$ & $1.65(1.25-2.16)$ & .003 \\
Sternal infection & $0.59(0.03-10.89)$ & .72 & $0.55(0.03-11.03)$ & .70 \\
Prolonged ventilation & $1.71(1.34-2.19)$ & $<.001$ & $1.54(1.21-1.96)$ & .001 \\
Renal failure & $2.00(1.07-3.75)$ & .03 & $1.91(1.09-3.35)$ & .02 \\
\hline
\end{tabular}

OR, Odds ratio; $C I$, confidence interval.

available to suggest that an organizational culture focused on quality might be more important than specific protocols in improving care. ${ }^{22}$ Each of these elements is prominent in the HeartSource model-monitored review of all outcomes in a risk-adjusted fashion using the highest standard of the profession (the STS database and benchmarks), external peer-review and open discussion of all deaths, significant complications, and interesting and/or challenging cases, shared protocols and educational opportunities, and emphasis on both process and outcome. It is not therefore surprising that surgical mortality has decreased with HeartSource involvement in each of the three programs in the present study that were not new programs.

The finding of an increased sternal wound infection rate, even risk adjusted in the LV programs, despite its statistical significance, must be viewed with a certain amount of caution, because only 6 patients in the entire study developed this complication. However, the greater rate of stroke in the large-volume programs, even after risk adjustment, is somewhat difficult to explain, but has been previously reported in a large study from the Nationwide Inpatient Sample. ${ }^{23}$ It, therefore, warrants additional investigation. Likewise, increased adherence to the use of the internal thoracic artery in larger programs was also noted in a large patient group from the STS database ${ }^{7}$ and might represent a greater percentage of nonelective cases in the LV centers.

\section{Study Limitations}

Although these results are certainly encouraging and supportive of a paradigm of care that integrates the academic medical center into the delivery of care at the community hospital level, the present study had numerous limitations that must be acknowledged. First, as a retrospective descriptive study, the results must be seen as hypothesis-generating rather than in any way definitive. The obvious absence of any control group did not permit us to distinguish or prove the specific causes for the results observed. The multifactorial and somewhat unique nature of CABG surgery might permit alternative explanations to those suggested. ${ }^{12}$ It remains unknown whether the excellent results achieved in our study could have been further improved by the addition of volume to these programs. Case selection was not monitored, and a choice to focus on low-risk cases would tend to make any differentiation in outcomes less apparent. Moreover, the effect of surgeon recruitment cannot be underestimated. In each case, the selection of surgeon was made by demonstrated clinical excellence, maturity, and appropriateness for academic appointment. It is therefore not unlikely that the "practice makes perfect" phenomenon as it relates to surgical experience was already ameliorated by the selection of seasoned surgeons to direct the programs.

Second, the small sampling of five hospitals did not permit definitive conclusions on a hospital level, although the patient level data were robust and were analyzed in a manner that accounted for clustering effects within centers. The potential effect of statistical variance in LV measurements ${ }^{3}$ was ameliorated by using data from multiple years. It is certainly notable, however, that two of the five hospitals had less than the STS recommended threshold of 100 cases/ year in each of the years measured, ${ }^{24}$ and none of the centers achieved the 450 Leapfrog benchmark in any year. Within this context, that the more commonly invoked parameters of hospital and surgeon volume ${ }^{25,26}$ had no relation to mortality or overall morbidity, but that adherence to quality measures did, is strongly suggestive that, at least in this context, the latter parameter is considerably more important in determining quality than the former.

Third, to evaluate a well-defined patient group with sufficient data to draw meaningful conclusions, our study only examined the results for CABG surgery. As has been previously noted, CABG surgery, as a well-developed and frequently performed procedure, might represent a unique case. ${ }^{12}$ Therefore, the applicability of these findings to valvular surgery, or other areas of cardiac surgery, remains to be explored.

\section{CONCLUSIONS}

In the setting of a university-based community hospital quality improvement program, excellent results can consistently be obtained in CABG surgery in relatively LV programs. The surgical outcomes in this context are not associated with program or surgeon volume but directly correlate with focus on quality as manifested by compliance with evidence-based quality standards. A meaningful university affiliation might represent a new quality paradigm for cardiac surgery in the community hospital setting. 


\section{References}

1. Luft HS, Bunker JP, Enthoven AC. Should operations be regionalized? The empirical relation between surgical volume and mortality. $N$ Engl J Med. 1979;301: 1364-9.

2. Birkmeyer JD, Siewers AE, Finlayson EVA, Stukel TA, Lucas FL, Batista I, et al. Hospital volume and surgical mortality in the United States. N Engl J Med. 2002; 346:1128-37.

3. Shahian DM, Normand ST. The volume-outcome relationship: from Luft to Leapfrog. Ann Thorac Surg. 2003;75:1048-58.

4. Shahian DM. Improving cardiac surgery quality-Volume, outcome process? JAMA. 2004;291:246-8.

5. Hannan EL, Wu C, Ryan TJ, Bennett E, Culliford AT, Gold JP, et al. Do hospitals and surgeons with higher coronary artery bypass graft surgery volumes still have lower risk-adjusted mortality rates? Circulation. 2003;108:795-801.

6. Post PN, Kuijpers M, Ebels T, Zijlstra F. The relation between volume and outcome of coronary interventions: A systematic review and meta-analysis. Eur Heart J. 2010;31:1985-92.

7. Shahian DM, O'Brien SM, Normand ST, Peterson ED, Edwards FH. Association of hospital coronary artery bypass volume with processes of care, mortality, morbidity, and the Society of Thoracic Surgeons composite quality score. $J$ Thorac Cardiovasc Surg. 2010;139:273-82.

8. Shroyer AL, Marshall G, Warner BA, Johnson RR, Guo W, Grover FL, et al. No continuous relationship between Veterans Affairs hospital coronary artery bypass grafting surgical volume and operative mortality. Ann Thorac Surg. 1996; 61:17-20.

9. Sollano JA, Gelifns AC, Moskowitz AJ, Heitjan DF, Cullinane S, Saha T, et al. Volume-outcome relationships in cardiovascular operations: New York State, 1990-1995. J Thorac Cardiovasc Surg. 1999;117:419-30.

10. Welke KF, Barnett MJ, Sarrazin MD, Rosenthal GE. Limitations of hospital volume as a measure of quality of care for coronary artery bypass graft surgery. Ann Thorac Surg. 2005;80:2114-9.

11. Rathore SS, Epstein AJ, Volpp KG, Krumholz HM. Hospital coronary artery bypass graft surgery volume and patient mortality, 1998-2000. Ann Surg. 2004;239: 110-7.

12. Shahian DM, Normand ST. Low-volume coronary artery bypass surgery: Measuring and optimizing performance. J Thorac Cardiovasc Surg. 2008;135: 1202-9.

13. Wilson CT, Fisher ES, Welch G, Siewers A, Lucas FL. U.S. trends in CABG hospital volume: The effect of adding cardiac surgery programs. Health Affairs. 2007;26:162-8.

14. Ricciardi R, Virnig BA, Oglivie JW, Dahlberg PS, Selker HP, Baxter NN. Volume-outcome relationship for coronary artery bypass grafting in an era of decreasing volume. Arch Surg. 2008;143:338-44.

15. Song HK, Diggs BS, Slater MS, Guyton SW, Ungerleider RM, Welke KF. Improved quality and cost-effectiveness of coronary artery bypass grafting in the United States from 1998 to 2005. J Thorac Cardiovasc Surg. 2009;137:65-9.

16. Hannan EL, Sarrazin MS, Doran DR, Rosenthal GE. Provider profiling and quality improvement efforts in coronary artery bypass graft surgery: The effect on short-term mortality among Medicare beneficiaries. Med Care. 2003;41: 1164-72.

17. Marcin JF, Li Z, Kravitz RL, Dai JJ, Rocke DM, Romano PS. The CABG surgery volume-outcome relationship: Temporal trends and selection effects in California, 1998-2004. Health Serv Res. 2008;43:174-92.

18. Hannan EL, Kilburn H, Racz M, Shields E, Chassin MR. Improving the outcomes of coronary artery bypass surgery in New York State. JAMA. 1994; 271:761-6

19. Grover FL, Johnson RR, Shroyer AL, Marshall G, Hammermeister KE. The Veterans Affairs continuous improvement in cardiac surgery study. Ann Thorac Surg. 1994;58:1845-51

20. O'Connor GT, Plume SK, Olmstead EM, Morton JR, Maloney CG, Nugent WC, et al. A regional intervention to improve the hospital mortality associated with coronary artery bypass graft surgery. JAMA. 1996;275:841-6.

21. Auerback AD, Hilton JF, Maselli J, Pekow PS, Rothberg MB, Lindenauer PK. Shop for quality or volume? Volume, quality and outcomes of coronary artery bypass surgery. Ann Intern Med. 2009;150:696-704.

22. Curry LA, Spatz E, Cherlin E, Thompson JW, Berg D, Ting HH, et al. What distinguishes top-performing hospitals in acute myocardial infarction mortality rates? Ann Intern Med. 2011;154:384-90.

23. Allareddy V, Ward MM, Allareddy V, Konety BR. Effect of meeting leapfrog volume thresholds on complication rates following complex surgical procedures. Ann Surg. 2010;251:377-83.
24. Clark RE. Outcome as a function of annual coronary artery bypass graft volume: the Ad Hoc Committee on Cardiac Surgery Credentialing of the Society of Thoracic Surgeons. Ann Thorac Surg. 1996;61:21-6.

25. Birkmeyer JD, Stukel TA, Siewers AE, Goodney PP, Wennberg DE, Lucas FL. Surgeon volume and operative mortality in the United States. $N$ Engl J Med. 2003;349:2117-27.

26. Zacharias A, Schwann TA, Riordan CJ, Durham SJ, Shah A, Papadimos TJ, et al. Is hospital procedure volume a reliable marker of quality for coronary artery bypass surgery? A comparison of risk and propensity adjusted operative and midterm outcomes. Ann Thorac Surg. 2005;79:1961-9.

\section{Discussion}

Dr Thoralf Sundt (Boston, Mass). Thanks, Paul, beautifully presented, and I would like to say thanks for getting your slides and your manuscript to me much earlier than I ever do for my invited discussants.

It is an intriguing hypothesis and, I think, an important topic. The volume/outcome issue seems to be a perennial topic, and it was even raised yesterday here in this meeting. Strangely, multiple studies have shown contradictory findings. It is much like the on-pump coronary artery bypass report that we just finished discussing, again a subject with many studies showing seemingly exactly contrary results. The report yesterday regarding heart transplantation suggested exactly the opposite of your work, with higher volume associate with better outcomes.

I would have to say that my reading is that volume is not a good surrogate for outcome. If we want to look at outcome, we ought to look at outcome. I think we are all agreed that organizations such as Leapfrog are looking under the wrong rock. If they want to look at outcome, they ought to look at meaningful outcomes.

But I think you go beyond this point and address another issue here-not just the question of whether or not volume is a good surrogate, but a second question, which is implied in your hypothesis-what does improve outcome, and, by inference, how can we intervene to improve those outcomes beyond concentrating cases in large-volume centers. It is a complex problem as it turns out. This same debate is going on in the general surgical community as they consider the introduction of the Surgical Care Improvement Project (SCIP) measures and the correlation with surgical site infections. The general surgeons are pulling their hair out, because they have seen a reduction in surgical site infections with the introduction of the program, but when they now consider the effect of each of the process variables one by one, they do not see good correlation with the outcomes.

But I think that you have hit on the answer, which is simply that it is the focus on process and the attention to outcome that is actually important. I suspect that the participation in a process improvement or quality-focused program amounts to "institutionalization" of the "Hawthorn Effect"- and, in fact, that might be a part of the explanation for the volume effect-that high-volume centers have, of necessity, improved process just by virtue of the actual quantity.

Although I agree with you up to this point, there are some issues that I do not understand about your analysis. It is certainly possible for an individual surgeon or system to focus on outcome, but not actually hit the mark on all variables despite their best efforts. What you have shown is that the patients in whom one meets the process variables-process variables that have been shown to correlate with good outcomes-have better outcomes. I could argue that this is circular logic. 
Instead, can we consider the parameters that might be surrogates for a focus on quality measures? In other words, can we stratify surgeons or institutions by their "batting average" in being consistent with the quality outcomes? Presumably, those with focus will hit the mark more often overall. Rather than looking at high-volume surgeons and low-volume surgeons, can we look at high-batting average surgeons and low-batting average surgeons and then see how their patients do? You wound up tracking the individual patients to the adherence to the metrics. What about highcompliance surgeons and low-compliance surgeons? So that is question number one.

Number two, did you have any surgeons that operated in two hospitals, two hospitals that might imply different systems factors such as a different focus on quality? So, for example, a given surgeon in hospital A might have better or worse outcomes in hospital $\mathrm{B}$ if the focus on quality at a systems level at that hospital is less.

Finally, what was the effect of the initiation of this program on adherence to those quality measures? Did a given program demonstrate better compliance with measures overall after joining the program?

I would like to emphasize what might have gone past some of the audience, because you went past it pretty quickly. This program is not one that was developed to drive cases to Columbia but one aimed at actually improving outcomes in the sites where you have intervened. It is altruistic in that regard. Thus, I believe that your work really answers the question that was posed during the heart transplant presentation yesterday: how can we improve quality in low-volume sites? Answer: Focus on quality.

Thanks. I really enjoyed your report very much.

Dr Kurlansky. Dr Sundt, thank you very much for your kind comments. Unfortunately, the statistical necessity did not permit us to effectively look at the hospital level or the surgeon level regarding their batting averages, because the models converge with so few centers; only five centers and only 1 to 2 surgeons per center. That is why we analyzed the data at the patient level. But it would certainly be very instructive as time goes and we generate more data to be able to track that.

We did not have any surgeons who operated at two hospitals in this program. In fact, none of the surgeons in this program operated at other hospitals. So we did not have access to try to assess that, although there have been some interesting studies, reports in the literature, regarding the effect of having, for example, a high-volume surgeon working in a low-volume program elsewhere and whether their results correlated more with the low-volume centers or the high-volume centers.

It is very difficult to be able to track exactly the effect of the program, because at some of these centers we helped them start the program. So there was not a program before. I can tell you this: in those centers in which there was a program before, in every case, we do know that the raw mortality declined. However, one of the aspects of this program was the institution of an information system that enabled us to track these sorts of results that did not exist before. So I cannot even retrospectively go back and find out what their performance measures were before we got there.

Dr Marc Ruel (Ottawa, Ontario, Canada). Dear Paul, I greatly enjoyed your presentation. I think, though, that there might be an issue here in terms of the statistical power of the study. When you are looking at dichotomous outcomes, power is not dependent on the denominator but on the numerator (ie, the number of events). For instance, with respect to mortality in your study, there were roughly 20 events of the 2000 or so patients whom you studied. So the power was fairly limited.

Therefore, I was wondering if you had the opportunity to calculate what would be the power of your conclusions? For instance, how certain are you and what power do you have to determine that you would, for instance, have identified a $40 \%$ relative increase in mortality in low-volume hospitals versus high-volume hospitals? Were you able to get this information out of your data?

Thank you very much for a great talk.

Dr Kurlansky. You raise an incredibly important point as pointed out yesterday so eloquently, that the absence of evidence is not evidence of absence, the classic problem of type II errors. And with only five centers, even with over 2000 patients, you have to account for clustering within centers, and therefore the power of this study to determine small differences or differences in low event rates is very limited.

However, what I think is extremely important here is that in that context where mortality and morbidity were not able to be distinguished, the power of compliance with National Quality Forum measures was extremely well statistically correlated with the difference in outcome, which tells me that even if there was a difference that with larger numbers would be measurable between the low-volume and the high-volume programs, that is not nearly as powerful a predictor as the National Quality Forum focus on quality compliance. Also, I think that is probably consistent with what was found with the review of the STS database that I alluded to where there are over a million patients, and there was a statistical correlation between outcome and volume, the degree to which volume contributed to the variance in the overall quality score was only $1 \%$. So, although it might be a predictor or it might actually be involved and we were not able to see it with the limited power of this study, I think that perhaps that limitation in power is a benefit to this study, because the fact that we were able to see some other parameter very powerfully highlights the point that process is much more important than volume in determining outcome. 
TABLE E1. Odds ratios of outcome measures in patients of lowversus high-volume hospitals adjusted for predicted risk and propensity for surgery in low- versus high-volume program

\begin{tabular}{lcc}
\hline \multicolumn{1}{c}{ Variable } & OR $(\mathbf{9 5} \% \mathbf{C I})$ & $\boldsymbol{P}$ value \\
\hline Mortality & $1.06(0.61-1.83)$ & .84 \\
Mortality or morbidity & $1.09(0.35-3.34)$ & .88 \\
Stroke & $0.27(0.15-0.46)$ & $<.001$ \\
Reoperation & $1.04(0.52-2.06)$ & .92 \\
Prolonged ventilation & $1.11(0.41-3.00)$ & .83 \\
Renal failure & $1.12(0.28-4.46)$ & .87 \\
\hline
\end{tabular}

Generalized estimating equation estimates for adjusted sternal infection analysis did not converge owing to small number of infections. $O R$, Odds ratio; $C I$, confidence interval.
TABLE E2. Odds ratios of outcome measures for patients with lowversus high-volume surgeons adjusted for predicted risk scores

\begin{tabular}{lcc}
\hline \multicolumn{1}{c}{ Variable } & OR $(\mathbf{9 5} \% \mathbf{C I})$ & $\boldsymbol{P}$ value \\
\hline Mortality & $1.60(0.76-3.37)$ & .22 \\
Mortality or morbidity & $1.13(0.78-1.64)$ & .51 \\
Stroke & $0.42(0.14-1.29)$ & .13 \\
Reoperation & $1.14(0.60-2.19)$ & .69 \\
Sternal infection & $0.66(0.11-3.99)$ & .65 \\
Prolonged ventilation & $1.10(0.64-1.90)$ & .73 \\
Renal failure & $0.96(0.46-2.03)$ & .92 \\
\hline
\end{tabular}

OR, Oodds ratio; $C I$, confidence interval. 